This is a Pre-Print version of the following article:

Luca Chiapperino, 'Epigenetics: Ethics, Politics, Biosociality', British Medical Bulletin 128, no. 1 (1 December 2018): 49-60, https://doi.org/10.1093/bmb/ldy033.

\title{
Epigenetics: ethics, politics, biosociality
}

\section{AUTHOR'S INFORMATION}

Luca Chiapperino

University of Lausanne, Faculty of Social and Political Sciences, Institute of Social Sciences, STSLab

Quartier UNIL-Mouline, Bâtiment Géopolis, CH-1015 Lausanne - Switzerland

Email: Luca.Chiapperino@unil.ch

Telephone: +41216923870

\section{STRUCTURED ABSTRACT}

\section{Background}

Epigenetics is a burgeoning field of contemporary biosciences, which has attracted a lot of interest both in biomedical and in social sciences

\section{Sources of data}

Unsystematic literature analysis and retrospective mapping of highly cited work (source: Web of Science core collection) in the social sciences and humanities engaging with epigenetics

\section{Areas of agreement}

Epigenetics poses no new ethical issue over and above those discussed in relation to genetics

\section{Areas of controversy}

However, it encourages a different framing and reflexivity on some of the commonly held categories in the moral uptake of scientific discoveries.

\section{Growing points}

Epigenetics presents us with normative questions that touch upon privacy, responsibility for individual health and for the wellbeing of future generations, as well as matters of health justice and equality of opportunities.

\section{Areas timely for developing research}

Epigenetic thinking could help us adjust and refine the problem-frames and categories that inform our ethical and political questions with a complex biosocial description of situations, of persons or actions

\section{KEYWORDS}

Epigenetics; privacy; responsibility; equity; biosocial 
Epigenetics: ethics, politics, biosociality - L.Chiapperino 


\section{Introduction}

The science of epigenetics is a burgeoning field of contemporary biosciences, which has attracted a lot of attention both in biomedical and in social sciences. Stricto sensu ${ }^{\mathrm{i}}$, epigenetics finds its origins in Conrad Hal Waddington's theory of epigenesis, pointing to the "need to remember that between genotype and phenotype, and connecting them to each other, there lies a whole complex of developmental processes" (p.10) ${ }^{4}$, which Waddington designated with the term 'epigenotype'. Currently, epigenetics represents a heterogeneous and interdisciplinary field of biosciences ${ }^{5}$, which thoroughly inscribes itself in the technologically intensive style of knowledge-production of the present post-genomic age. First, the field of epigenetics encompasses some research endeavours that, after the completion of the Human Genome Project, are directed to the establishment of profiles and functional maps of our genome, by resorting to next-generation sequencing technologies. Epigenomics aims at producing complete cartographies of the specific chemical modifications of DNA (e.g. methylation), histones (e.g. histone methylation, acetylation, phosphorylation), chromatin (e.g. nucleosome positioning), and the RNA expression profiles that produce specific states of our genome when differentiating into the several cellular types of our bodies at different stages of development, and in pathological conditions ${ }^{6,7}$. Second, numerous studies have accumulated on the role of the epigenome as a biological interface for the embodiment of social conditions, biographical histories, and environmental exposures. In this respect, epigenetics represents a knowledge repertoire that promises to account for the 'molecular conduit' ${ }^{8}$ that links one's milieu and life conditions with one's basic biological functioning. For this reason, epigenetic methods and techniques have productively engaged with long-standing epidemiological questions about the relationship between social status and health over the lifecourse, the role of developmental processes and infant's early life in the programming of adult health, or even the effects of long-term exposures to chemicals in the aetiology of the most common diseases ( $\operatorname{see}^{9}$ for an overview). Third, epigenetics includes also studies accounting for different temporal scales of those processes of embodiment and biological archival of one's experiences. Several animal studies, and some human studies have produced evidence of multigenerational transmission of epigenetic marks acquired by the parental generation related to metabolic predispositions, as well as behavioural traits (e.g. stress coping, depression) ${ }^{10}$. These enticing and at times contested findings ${ }^{11}$ highlight the possibility of gamete-mediated transmission of traits epigenetically acquired during the lifetime across multiple generations, and

\footnotetext{
${ }^{\mathrm{i}}$ It should be noted that the research themes and issues currently at the centre of epigenetic sciences have a much longer history in that they go at the core of questions related to the plasticity of our bodies in ontogenesis, the permeability of biology to environmental and social conditions during the lifecourse, as well as the bearing of these processes in adaptation, inheritance and evolution; see ${ }^{1,2,3}$.
} 
postulate the importance of understanding individual predispositions as standing in continuity with the health, biographies, life conditions and actions of both previous and following generations.

The present review aims at presenting an overview of the ethical, political and social aspects of epigenetics, drawing from an unsystematic literature analysis and retrospective mapping of highly cited work (source: Web of Science core collection) in the social sciences and humanities engaging with this domain of the life sciences. As we will see below, the theoretical and experimental perspectives animating epigenetics render this field a particularly prolific terrain of engagement for social sciences and the humanities, and raise challenges that go beyond the mere apportionment of normative exercises at the level of individual and/or collective agencies. Epigenetic knowledge does not only present us with ethical and political issues of utmost relevance, but rather seems to encourage a different framing and reflexivity on some of the commonly held categories in the moral uptake of scientific discoveries.

\section{Ethical normativity}

It is worth noting here how institutional configurations and analytic categories deployed for the societal uptake of epigenetics owe much to the advent of genetics, as well as to its scrutiny on the side of social sciences and humanities scholarship. The praxis of the ELSI program established within the Human Genome Project ${ }^{12}$ has in fact provided a model upon which to build several international consortia also in epigenetic science (e.g. The International Human Epigenome Consortium), which have endowed themselves with similar working groups on the ethical implications of their research. These bioethicists play a vital role both in establishing and mitigating the ethical issues internal to the proceedings of scientific practices (e.g. providing guidance to data and sample handling, establishing privacy protection standards specific to epigenetic research), and in contributing to the analysis of the potential ethical challenges emerging from epigenetic science.

Chiefly, however, the close engagement of these scholars with the practical configurations of epigenetic science, modelled on the scholarly scrutiny of science established in genetic research, has produced the significant centrality of the question: is epigenetics confronting us with any exceptional issue that was not raised by genetics ${ }^{13}$ ? With regard to this point, a substantial agreement exists in the bioethical literature. Epigenetics, according to several scholars, poses no "new ethical issue over and above those discussed in relation to genetics" (p.469) ${ }^{14}$; however - as we will see in the remaining it complicates the established framing and intensifies the cogency of several of them.

\section{Privacy and confidentiality}

The first issue is the one of privacy and confidentiality of health-related information. Epigenetics is in fact another instance of the broader problem of how to ensure an appropriate protection of sensitive 
data from research participants and patients at a time in which vast networks of *omics research data and health records are being created worldwide. Yet, epigenetic data appear to intensify the ethical concerns attached to risks of privacy breaches and re-identification in two main respects. Primarily, due to the fact that methylation study techniques like Whole-Genome Bisulfite Sequencing (WGBS) could expose genetic information, and thus potentially lead to re-identification of sample donors. As shown by Dyke and colleagues ${ }^{15}$ public epigenomic databases report DNA methylation data that are indirectly affected by single-nucleotide polymorphisms (SNPs), and consequently provide an information specific to individual participants that could potentially be used for re-identification through genetic matching. By making accessible epigenetic data, epigenomic databases thus run the risk to expose also genetic information which normally would not be made accessible. Although the removal of this direct genotype information does not ensure absolute privacy, these scholars have called for the development of policies specific to epigenetic data release in major international consortia that could reasonably minimise the risks of re-identification. Secondly, epigenetics raises privacy-related concerns for the possibility to impute personal data from methylation encompassing not only medical conditions (e.g. disease predispositions), but potentially also lifestyle behaviours. Although Yann Joly and colleagues rightly warn us that we should not inflate the (limited) combined risks of re-identification and privacy breaches from epigenetic data ${ }^{16}$, validated studies have so far showed that sensitive information such as alcohol and tobacco consumption information can be accurately inferred from methylation arrays ${ }^{17}$. It is thus possible to speculate that, as evidence of epigenetic signatures for substance-consumption, experiences and lifestyle behaviours increases, the risks of privacy breaches conditional to re-identification of donors from epigenetic data are also likely to intensify, and could lead to secondary uses of epigenetic information in various settings such as insurance (e.g. distinguishing never, former or current smokers) and employment (e.g. establishing an employee's susceptibility to disease $)^{13}$. This possibility has led also to the call for legislative updates of current regulations preventing genetic discrimination, as a substantive disagreement exists concerning their applicability to epigenetic data ${ }^{18}$. Finally, speculations ${ }^{19}$ about a potentially positive role of these epigenetic markers of lifestyles and individual experiences can also be found with regard to their potential use for forensic purposes. These epigenetic bio-social archives could in fact allow, according to this stream of work, the inference of both behavioural information (e.g. alcohol and tobacco consumption) and bodily features (e.g. biological age) of the person to which the samples recovered on a crime scene belong.

\section{Epigenetic responsibility for health}

The capacity of epigenetic signatures to account biologically for how individual behaviours and lifestyles affect the phenotype later in life (and potentially across generations) leads us to the second 
ethical controversy substantively revamped by epigenetics: individual responsibility for health. Several scholars have in fact already warned us against the possibility that epigenetic knowledge may be presented as making visible, at the molecular level, the consequences of individual unhealthy behaviours ${ }^{20,21}$. Precisely by making knowledgeable the effects of our habits on our health, these authors argue, epigenetics may become an enabling platform to anchor normative options of 'what ought to be' done with regard to one's health down to the level of the 'integrity' of our epigenome. Epigenetics is attributed the potential to go beyond correlative epidemiological data about risky behaviours and their consequence for health, and thus could constitute knowledge of the molecular chain of events that starts from a certain lifestyle and/or environmental factor and causally leads to disease development. Being framed as accountability for damaging, or as prospective responsibility to improve one's own epigenome, several authors agree that the molecular quantification of the effects of lifestyles on our health could problematically "make a change in degree" (p.178) ${ }^{21}$ in moral claims of individual versus collective responsibilities for health, or at least constitute an evidencebase for compelling individuals "to do something" (p.464) ${ }^{14}$ for protecting it. Furthermore, this feature of epigenetics may also constitute the epistemic correlate and evidentiary legitimisation of the well-established policy emphasis on responsibility for health, often resorting to rhetorics of empowerment and self-determination with regard to health ${ }^{22}$.

The reasons to be wary of claims to epigenetic responsibility for health rest primarily on empirical grounds; namely, the epistemic and actionable value of the correlations between epigenetic markers and lifestyles. As shown by prominent epigenetic epidemiologists Bastiaan Heijmans and Jonathan Mill ${ }^{23,24}$, the characterization of the effects of individual habits - but also exposures, life conditions, psychosocial factors - on the epigenome is limited in several respects. First, the correlations between epigenetic marks and environmental stimuli have yet to prove their biological implications in terms of gene expression, function, and consequently their causal role in pathogenesis. It is in fact far from being established whether changes in (for instance) DNA methylation translate into differences in gene expression in a disease-relevant manner, whether these changes are individually responsible for the phenotypes observed, or whether they may affect gene transcription only in functional networks (when they co-occur with other changes to gene expression, due for instance to other independent modifications of the methylome). Second, epigenetic variation is not due solely to environmental stimuli such as lifestyle behaviours. As it is known, epigenetic marks are malleable and reversible and respond to several other factors in the external milieu, as well as those in the internal cellular environment due to (for instance) individual genetic differences. Adjudicating whether an effect is due to lifestyles, other environmental stimuli, or genetic differences - or indeed a combination of the three - is thus a complex puzzle that is currently impossible to solve, on the basis of the available 
technologies and knowledge. Besides this, it should also be noted the role of stochastic processes in the epigenetic patterning of gene expression in different cell types.

The observation that both deterministic and stochastic factors can shape epigenomic variation suggests therefore that epigenetic studies may be unable to disentangle respectively the causal role of individual lifestyle-related, genetic and environmental factors in the aetiology of disease, and consequently constitutes a strong empirical reason to normatively dismiss the claims to epigenetic responsibility introduced above. As argued by Hedlund ${ }^{21}$, these empirical observations against causal individual responsibility for the aberrant effects of epigenetic variations shift the normative burden of prevention to collective agencies (e.g. the State, public health agencies). Since individual factors act in concert with social, economic, political structural conditions - and, it should be added, also other material determinants such as genetic variation - responsibilities for epigenetic predispositions to diseases should not be handled individually. The causality behind lifestyle-related diseases, she argues, is an intricate web of social, economic and political factors of which no individual could be ascribed full responsibility.

Other scholars have called instead for a more nuanced approach to epigenetic responsibility, which would not limit itself to alternatively locate it at the level of individual or collective agencies. Dupras and Ravitsky ${ }^{25}$, for instance, dismiss individual responsibility claims in light of the complex spectrum of plasticity, variability and indeterminacy characterising our epigenome. If the epigenome changes over temporalities of development, over individual genetic differences, and stochasticity - they argue - what healthy behaviours should individuals strive for to avoid aberrant epigenetic modifications in a morally relevant sense? Yet, the authors also disagree with Hedlund in that they consider assigning responsibility only to States and collectives to be simplistic. According to them, denying that some consequences of aberrant epigenetic effects may fall under the scope of individual agency - provided that certain conditions of capacity to act are met, and that action takes place in a time window of susceptibility for a specific and known epigenetic effect - means misrepresenting the complex nature and opportunities offered by epigenetics. For this reason, Dupras and Ravitsky call for a normative uptake of epigenetic responsibility that is attentive to scientific detail and limitations and that establishes the (material and moral) conditions as well as opportunities to act effectively in order to protect one's epigenome.

Furthermore, several Science and Technology Studies (STS) scholars have rather focused on the socio-political conditions behind the production and recruitment of epigenetic knowledge in support of normative claims of individual responsibility for health. While Ruth Müller and her colleagues ${ }^{26,27}$ have highlighted from an interdisciplinary perspective how the language and metaphors of reductionism and determinism (e.g. an alleged epigenetic mark for poverty) in biological sciences are 
one reason for such simplistic normative translations of epigenetics, other authors have instead focused on the co-production of epigenetic knowledge of lifestyle-related effects and current social discourses, policy frameworks and political cultures of individualization in healthcare. Epigenetics is - to this latter view - not just contributing evidence in support of responsibility-sensitive policies for future healthcare. Rather, the kinds of questions and answers animating this field of research are one result of the socio-political emphasis put on the devolution of power and responsibility for health to individuals ${ }^{22}$. This shared recognition calls for further empirical investigations into the hidden assumptions and premises of knowledge-production in epigenetics, which could highlight the narratives, metaphors and moralizing frameworks informing research into the molecular effects of lifestyles and behaviours.

\section{Parental and procreative epigenetic responsibility}

Evidence of multigenerational epigenetic effects purport that parental biological influences extend past the moment of conception, thus assigning a pivotal role to pre-conception pathways of inheritance of biological predispositions. From effects of parental (and also grandparental) exposures, to in utero signalling and transduction of environmental influences, the picture drawn by this stream of epigenetic research suggests extending arguments in favour of the 'responsibility for protecting one's epigenome' to the context of reproduction.

Needless to say, responsibilities of prospective parents in reproductive practices are no novelty brought about by epigenetics. Boosted by the advent of pre-implantation genetic diagnosis (PGD), and various prenatal genetic diagnostic or screening techniques, sociological and philosophical scholarship has widely emphasized the creation of new normative and societal domains in reproduction. Some authors have focused on the ethical and political issues raised by the possibility to select and intervene upon the child's genetic make-up ${ }^{28}$. Other scholars have instead focused on the mutual shaping of reproductive technologies and the social order of procreative practices, by highlighting the generation of a 'biomedical mode of reproduction' ${ }^{29}$. Within this context, the discourse emerging from multigenerational epigenetics may - once again - reframe normative issues related to the responsibilities we hold to promote the health of future generations. In particular, epigenetic knowledge of how lifestyles and environmental hazards are likely to yield adverse health outcomes in the offspring and in subsequent generations intervenes upon these matters in three main respects. First, the legacy of epigenetic marks may expand the temporal boundaries of already disputed parental obligations to 'procreative beneficence'; i.e. the idea that we might have a moral obligation (where a choice can be made) to bring to birth the "best" child possible, or at least to minimize her potential suffering ${ }^{30}$. By shedding a light on the potential harmful consequences of individual unhealthy lifestyles (from birth to reproduction) on future generations, epigenetics may 
extend these controversial normative claims far beyond the moment of reproduction, thus compelling individuals to mitigate the risks and threats to the wellbeing of those we (will) care for over the whole life-course. From recasting young adults as responsible prospective parents (even in the absence of any imminent project of parenthood), to instating liability of actual parents for past imprudent behaviours, these epigenetic data fold different temporalities ${ }^{31}$ into moral claims to procreative beneficence. Acting responsibly towards the health of future generations would include, if we take at face value studies of epigenetic inheritance, predispositions acquired by prospective parents throughout their whole life-course, and would extend also to those they have inherited from their own parental lineages. Second, this focus on pre-conception pathways of transmission of disease predispositions touches upon the distribution among the prospective parents of normative claims related to lifestyle-related epigenetic programming of health and disease. Although several social scientists have compellingly shown a systematic moral emphasis on effects transduced by the mother during pregnancy ${ }^{32,33}$, evidence of epigenetic transmission through the male germline adds the normative relevance of non-genetic predispositions inherited from the paternal line to claims of responsibility for health in light of future generations. The growing evidence of paternal effects is in fact vocalised within the community of scientists - but perhaps then not much put to action - as encouraging researchers to think about the developmental contribution of both parents equally, or at least to acknowledge the differential contribution of maternal and paternal epigenetic predispositions, along with the gestational period, when looking at the epigenetic origins of adult diseases. Third, evidence of epigenetic multigenerational effects constitutes a moral imaginary of responsibility to protect the wellbeing of future generations that, in contrast to genomics, appears to fall within everyone's means ${ }^{21}$. Knowledge of epigenetic inheritance does not require in fact its crystallization in the form of a technology (e.g. prenatal testing, pre-implantation genetic diagnosis) to demand actionability mainly because it enlarges the scope of responsibility for the health of (future) offspring to cover the requirement to adopt a healthy lifestyle for both prospective mothers and fathers. This element has led some scholars to highlight the risk that the prospect of these studies may be translated into prescriptive messages for parents-to-be well before reaching its time as established and validated medical knowledge ${ }^{34}$.

Obviously, we will not reiterate here how the above-mentioned arguments against claims to epigenetic responsibility tout court apply also in the case of studies focusing on the multigenerational transmission of epigenetic predispositions to disease. Claims to epigenetic parental responsibility are in fact problematic for the same reasons encountered above. On the one hand, they diverge the attention from the complex causality of disease development and the corresponding collective responsibilities to mitigate detrimental structural effects of social arrangements and determinants of 
health on periconceptual, prenatal, childhood and adolescent development. On the other hand, it should also be noted how studies of epigenetic multigenerational effects are often recruited as a "solid" epistemological and technological ground to confirm existing social emphasis and prejudices, and in particular those upon the body of the mother as a locus of political government of health, and prevention of future diseases ${ }^{32,33}$. Yet, also other counter-arguments that are specific to the intergenerational nature of these effects apply to claims in favour of parental and procreative epigenetic responsibility.

The first of such lines of criticism argues that procreative epigenetic risk messaging could pave the way to what Juengst and colleagues ${ }^{34}$ have termed 'epi-eugenics'; that is, an undue ${ }^{\mathrm{ii}}$ social pressure on prospective parents to limit preconception and prenatal risky exposures, or to undergo testing for epigenetic alterations in the gametes and/or developing foetus. Very much like in the case of genetic technologies - which have been deemed problematic for their encouragement of practices of selection and optimization in reproduction - one reason for thinking of claims to parental and procreative epigenetic responsibility in negative moral terms stems from the condemnation attached in common sense morality to the eugenic imperative of tinkering with and improving the human biological pool. With the addition, however, that - as shown above - the complexity of the epigenome calls into the question the capacity of individual actions to fulfil this imperative in the face of larger social configurations having an effect on epigenetic predispositions, and thus runs the risk of making prospective parents responsible for what should be collective public health concerns.

This leads us to a second argument against parental and procreative epigenetic responsibility, which points to the societal reverberations of the injunction towards the protection and optimization of epigenetic predispositions for the benefit of future generations. According to this view, what is wrong with these practices is that they send out a message about existing people: either people (and their parents) with specific conditions due to an epigenetic mark, or all those who (for instance) share a specific life history or socioeconomic status. Nourishing expectations for prospective parents to prevent these effects might unfairly target the most vulnerable groups in our societies, and create the condition for social blame of those already suffering from injustices. As summarized by Juengst and colleagues $^{34}$, promoting parental and procreative epigenetic responsibility for migrants exposed to

\footnotetext{
ii I will not delve here into definitional issues of 'eugenics', which also impact its evaluation in moral terms. It is in fact far from obvious that any practice falling under a descriptive definition of 'eugenics' such as 'improving the human biological pool' can be deemed morally problematic. Yet, it suffices to say for the purpose of the present analysis that the use of the term by Juengst and colleagues is clearly connoted with condemnation, although the criteria for holding such practices problematic are not specified in their work. For a socio-historical analysis of both reactionary and progressive political stances in the eugenic movement, see chapter $3 \mathrm{in}^{3}$. For an analysis of the moral reasons and arguments to hold eugenic practices morally problematic, see chapter $6 \mathrm{in}^{28}$.
} 
pesticides, or those living around food deserts, or those exposed to personal and collective tragedies "merely adds insult to moral injury" (p.428).

Third, one of the main critiques of future-oriented responsibilities to protect one's epigenome is the famous objection known as the non-identity problem. This long-standing philosophical issue, which is particularly cogent in relation to transgenerational epigenetics ${ }^{35,36}$, starts from the recognition that actions undertaken for protecting future generations will impinge on the number and identity of people that will be brought to existence. This means that both acting and not acting to do so cannot be said to benefit or harm anyone simply because these two lines of action engender two different sets of people, who both owe their existence precisely to the choice in question. Thus, especially in the case of epigenetic effects transmitted through the gametes - i.e., effects that do not affect a given existing individual, such as those transmitted from grandparents to grandchildren - claims in favour of procreative and parental epigenetic responsibility appear to fall into a philosophical paradox. Whose benefit would be promoted by acting responsibly to protect one's epigenome for the sake of future generations' wellbeing? Unless we are facing an actual individual, such as in the case of gestational epigenetic effects ${ }^{37}$ - which are however problematic for their targeting of maternal bodies - the objection from the non-identity problem suggests that a failure to fulfil such responsibility cannot harm future generations. Furthermore, this issue finds an additional complication when we take into account that epigenetic predispositions assume a positive or negative health-related value only when certain environmental conditions are met. In other words, some epigenetic marks (e.g. metabolic predispositions) may turn out to be potentialities that get realized as pathological (e.g. cardiovascular disease) only in the mismatch with a given social context (e.g. food availability and composition $)^{38}$. How to establish then the right actions to undertake in the present when the living conditions of future generations may be different?

\section{Political normativity}

So far, we have encountered critiques to the normative exercises stemming from epigenetic knowledge that argued for the need of addressing these issues at a collective and political level. The complexity of epigenomic programming of health points in fact to the combined contribution of individual agency and structural social conditions in these processes, and thus suggests the need to adopt a public health and/or populational perspective to justify the moral cogency of interventions based on epigenetic knowledge. In this section, I will explore in more details how epigenetics relates to political arguments touching upon notions of health equity and equality of opportunities; once again, as we will see, epigenetics does not present us with new issues, but rather seems to intervene upon the framing and the established categories employed to address questions of social and 
distributive justice in healthcare. Instead of focusing, then, on the potential evaluation of epigenetics from the standpoints of distinct theories of justice in healthcare ( $\mathrm{cf}^{39,40}$ ), here I will focus on whether epigenetics substantively reconfigures the moral exercises required by these approaches to justice in health.

\section{The shift from risk groups to individuals at risk}

One of the controversies around epigenetics in health justice matters has to do with a shift in perspective when evaluating the unequal distribution of harmful exposures in our societies. Substances that cause aberrant epigenetic effects - such as pesticides, pollutants, toxic chemicals, bad nutrients, tobacco - are in fact not distributed equally in the general population. Being exposed to such substances is often associated with socioeconomic position, or with broader societal configurations (e.g. occupational exposures, living conditions, food deserts), which have already been shown to frequently burden the worst-off in our societies more than the average population. As emphasized by the long-standing epidemiological literature on the social determinants of health ${ }^{41}$, promoting social mobility and reducing these broader inequalities might in fact have an aggregate impact on health greater than an approach at level of individual care. So, the question arises: is epigenetics simply reiterating the same normative claims to correct these inequalities (but on different epistemic grounds), or is this knowledge heightening moral obligations to remediate these risks? In answering this question, different authors appear to take (at least) two distinct positions. On the one hand, Rothstein and colleagues ${ }^{42}$, and Huang and $\mathrm{King}^{43}$ point out that epigenetics does not add anything sufficiently unique to the question of health inequalities, as a matter of ethics, policy and regulations. Harms due to the skewed distribution of exposures, societal configurations, and their multigenerational effects are no novelty in the biomedical literature, and may also be caused by biological mechanisms other than epigenetics. Genetic somatic mutations, Rothstein and colleagues argue, are also following a similar distributive pattern in our societies, and yet they have not produced any significant impetus to correct environmental injustices. Thus, Rothstein and colleagues ask, "is it logical or politically expedient to focus on the biological basis of the harm?” (p.7). Furthermore, these authors also find reasons not to invest epigenetic mechanisms with such an expectation. In a society characterised by indifference to "the plight of the less fortunate", and by a reductionist emphasis on the internal causes of disease, there is a risk that the societal response to epigenetic harms will privilege "clinical rather than environmental interventions to treat those afflicted", thus brushing over the structural "social and economic causes of hazardous exposures" (p.8). On similar grounds stands the critique of Huang and King ${ }^{43}$. These authors add to arguments against the exceptionality of epigenetics for social justice also a fine-grained critique of the inexpedient nature of epigenetic marks to support policies for the improvement of environmental conditions. As shown by the 
literature supporting the 'mismatch hypothesis' 38 , they argue, epigenetic predispositions to health disadvantages depend very much on the context: some patterns of epigenetic programming are maladaptive, or represent alternatively an improvement of individual fitness only conditionally to a given environment. Thus, the formulation of universal policies against a specific epigenetic mark may be impossible, if not worth avoiding on moral grounds, in order not to prevent the benefits of certain predispositions to arise under the local conditions that render them so.

On the other hand, different authors ${ }^{40,44}$ emphasize how epigenetics could concretely measure the magnitude of biological insults due to environmental exposures, and consequently quantify the harm caused by health inequalities resulting from social arrangements in a given individual. The specificity of epigenetics thus amounts to its possibility to deliver "health monitoring markers" (p.147) ${ }^{40}$ that could identify the aberrant impact of social background on health on the basis of considerations of time (e.g. sensitive windows), context (e.g. concurring environmental conditions) and biological background (e.g. individual genomic variance). Starting from this recognition and from a luckegalitarian perspective ${ }^{40}$ (i.e. the idea that we have a duty to compensate inequalities that are due to social and biological factors beyond one's control), it could be argued that the possibilities offered by epigenetics for health justice consist in a finer prioritization of resources correcting inequalities beyond individual control. Primarily, because epigenetics would offer a tangible measure of the increased risk to which some disadvantaged individuals are exposed, simply due to bad luck (e.g. social position, upbringing, living conditions). Epigenetics might therefore at least aid to quantify the impact of long-term accumulation of environmental insults on people from disadvantaged social background. Secondly, because stratification of individual risk on an epigenetic basis would even allow equalizing the health outcomes in the known risk groups, as it could enable the development of prevention strategies directed to those - more unlucky among the unlucky, it could be said - who are more likely to develop a disease based on a combination of physiological and environmental factors. In other words, knowledge of epigenetic mechanisms may not only confirm the moral necessity - for those who hold it - to intervene upon the skewed distribution of environmental hazards and health risks in certain groups of our societies, but could potentially shift questions of justice from "should we correct the unequal distribution of exposures in a society", to "who are those individuals harmed by such unequal conditions"?

\section{Blurring the boundary between natural endowments and social inequalities}

A second controversy concerning the impact of epigenetics on justice-based evaluations of healthrelated inequalities deals with qualification of epigenetic predispositions - especially those acting upon multigenerational temporalities - as natural endowments, or as effects of social position acting via a biological mechanism. In fact, understanding epigenome-based health disadvantages as one or 
the other has also an impact over their evaluation as unjust social inequalities. As shown by different authors $^{39,40,44}$, the categories pertinent to distinct theories of justice may provide a limited framework from which to construct an evaluation of the complexity of epigenetic processes in terms of health equity. To illustrate this point, let us refer to one example of this difficulty highlighted by Loi and colleagues $^{40}$ in relation to Rawlsian egalitarianism. In Rawlsian terms, there is an obligation to remedy to unequal opportunities if these are not due to natural endowments, i.e. biological predispositions and talents possessed at birth that are distributed through a "natural lottery". Also known as the Fair Equality of Opportunity (FEO) principle, this bit of Rawls' theory of justice demands that no social or legal barrier shall be put over the possibilities to improve one's social position, and that people who are endowed with the same talents and motivations should have equal chances of achieving these positions. In a nutshell, Rawlsian egalitarianism places the predispositions we are born with outside the scope of justice. How then to evaluate epigenetic disadvantages on the basis of the FEO principle? Loi and colleagues ${ }^{40}$ provide a mixed answer to this question. One line of reasoning could indeed point out that the epigenetic predispositions people are born with are just another example of the natural "(epi)genetic lottery" (p.149) ${ }^{40}$ whose correction does not concern the Rawlsian egalitarian. If indeed we define these epigenetic marks as natural endowments possessed at birth, then promoting the FEO principle does not demand to correct them, regardless of whether they result from social inequalities. A different line of reasoning could instead point out how epigenetic marks are nothing more than a social inequality acting through a biological mechanism, and thus conclude that they require the application of the FEO principle. Indeed, epigenetic risks and predispositions are sensitive to the starting social position of an individual in the case of gestational effects, or even the result of the adverse and disadvantaged social conditions experienced by parents and grandparents in cases of multigenerational epigenetic inheritance.

Without the need to solve this philosophical and normative quandary $\left(\mathrm{cf}^{45}\right)$, it is worth underlining once again - how the example of Rawlsian egalitarianism offers an illustration of the challenges raised by epigenetics to the established categories for the normative assessment of health inequalities. More than requiring the application of theories of justice for the resolution of its societal issues, epigenetics seems to question commonly held views about what counts as natural and/or sociopolitical (inequality) and blurs the distinction between disadvantages due to natural endowments and those due to social, economic and political structures. In other words, epigenetics provides a clearcut example of how the biology of individuals is partly a social factiii: how does this affect the questions we can ask about the good of our societies?

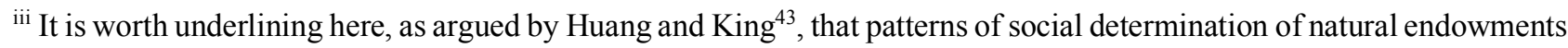
are in no way an exceptional specificity of epigenetics. Without the need to emphasize how alternative biological pathways of embodiment of social conditions currently populate the landscape of post-genomic biosciences ${ }^{46}$ (e.g.
} 


\section{Conclusions: epigenetics, biosociality, moral thickness}

At the core of the epigenetic endeavour lies the attempt to account for the social, political, economic and culturally-situated unfolding of our biology. This movement from the life sciences towards a social view of biological facts offers a seemingly promising avenue to rethink the aetiology of common diseases, their distribution in our societies, as well as the role of individual/collective agencies across multiple temporal frames, life contexts, and socio-political configurations. In a nutshell, epigenetics offers the opportunity to conceptualize what Meloni and colleagues ${ }^{46}$ explore as the biosociality of human life; namely, "the fundamental continuity of biological and social forces, organism and environment, agent and milieu" (p.5).

In this review, I have briefly summarized how these biosocial openings in epigenetics interrogate us not just in terms of normative options, but also in terms of moral thinking. Epigenetics presents us with normative questions that touch upon privacy, responsibility for individual health and for the wellbeing of future generations, as well as matters of health justice and equality of opportunities. Yet, before deploying any evaluative outlook on these matters, the ideas, concepts and evidence animating the field of epigenetics also demand an engagement with present and past conditions of individuals (and their ancestors), or the estimate of people's material and social opportunities to prevent and/or correct their aberrant predispositions, or a description of the socio-political and cultural realities they embody through epigenetic mechanisms. In doing so, epigenetics encourages therefore thickening moral exercises of privacy, responsibility, justice, and equity with a complex biosocial description of situations, of persons or actions, in order to afford their significantly balanced evaluation. The way these moral notions can be used in the case of epigenetics is, in other words, determined by temporalities, types and scales of embodiment of the social world, which urge us in turn to adjust and refine, in a situated manner, the problem-frames and categories that inform our ethical and political questions as well as judgements.

microbiomics, metabolomics), the authors rightly underline how "any form of selection of traits due to parental advantage, [...], would serve to undermine the concept of randomly distributed "natural endowments" (p.77; original emphasis). In this respect, epigenetics may thus be regarded as no more than a cogent example of these broader dynamics, and one that may produce some biological signatures of embodied social disadvantages. 


\section{Acknowledgements}

This work is part of the Swiss National Science Foundation (SNSF) project "PaRED-Parental Responsibilities, Epigenetics and DOHaD” (Project $\mathrm{N}^{\circ} 162873$ ). The author would also like to acknowledge - as founding learning experience useful to the preparation of this paper - his previous doctoral engagement with the "Dissemination and Healthcare Policy" program of the Italian National Research Center (CNR) Flagship Project EPIGEN.

\section{Bibliography}

1. Jablonka, E. \& Lamb, M. J. Evolution in four dimensions: genetic, epigenetic, behavioral, and symbolic variation in the history of life. (MIT Press, 2005).

2. Fox Keller, E. The Mirage of a Space between Nature and Nurture. (Duke University Press, 2010).

3. Meloni, M. Political Biology. (Palgrave Macmillan, 2016).

4. Waddington, C. H. The Epigenotype. Int. J. Epidemiol. 41, 10-13 (2012).

5. Tolwinski, K. A new genetics or an epiphenomenon? Variations in the discourse of epigenetics researchers. New Genet. Soc. 32, 366-384 (2013).

6. Roadmap Epigenomics Consortium et al. Integrative analysis of 111 reference human epigenomes. Nature 518, 317-330 (2015).

7. Stricker, S. H., Köferle, A. \& Beck, S. From profiles to function in epigenomics. Nat. Rev. Genet. 18, 51-66 (2016).

8. Landecker, H. \& Panofsky, A. From Social Structure to Gene Regulation, and Back: A Critical Introduction to Environmental Epigenetics for Sociology. Annu. Rev. Sociol. 39, 333-357 (2013).

9. Epigenetic Epidemiology. (Springer Netherlands, 2012).

10. Szyf, M. Nongenetic inheritance and transgenerational epigenetics. Trends Mol. Med. 21, 134-144 (2015).

11. Daxinger, L. \& Whitelaw, E. Transgenerational epigenetic inheritance: More questions than answers. Genome Res. 20, 1623-1628 (2010).

12. Thomson, E. J., Boyer, J. T. \& Meslin, E. M. The Ethical, Legal, and Social Implications Research Program at the National Human Genome Research Institute. Kennedy Inst. Ethics J. 7, 291298 (1997).

13. Rothstein, M. A. Epigenetic exceptionalism. J. Law Med. Ethics J. Am. Soc. Law Med. Ethics 41, 733-736 (2013).

14. Chadwick, R. \& O’Connor, A. Epigenetics and personalized medicine: prospects and ethical issues. Pers. Med. 10, 463-471 (2013). 
15. Dyke, S. O. M. et al. Epigenome data release: a participant-centered approach to privacy protection. Genome Biol. 16, 142 (2015).

16. Joly, Y., Dyke, S. O., Cheung, W. A., Rothstein, M. A. \& Pastinen, T. Risk of re-identification of epigenetic methylation data: a more nuanced response is needed. Clin. Epigenetics 7, (2015).

17. Philibert, R. A. et al. Methylation array data can simultaneously identify individuals and convey protected health information: an unrecognized ethical concern. Clin. Epigenetics 6, 28 (2014). 18. Rothstein, M. A., Cai, Y. \& Marchant, G. E. The Ghost in our Genes: Legal and Ethical Implications of Epigenetics. Health Matrix Clevel. Ohio 1991 19, 1-62 (2009).

19. Relton, C. L., Hartwig, F. P. \& Smith, G. D. From stem cells to the law courts: DNA methylation, the forensic epigenome and the possibility of a biosocial archive. Int. J. Epidemiol. 44, 1083-1093 (2015).

20. Rothstein, M. A. Legal and Ethical Implications of Epigenetics. in Environmental Epigenomics in Health and Disease (eds. Jirtle, R. L. \& Tyson, F. L.) 297-308 (Springer Berlin Heidelberg, 2013).

21. Hedlund, M. Epigenetic Responsibility. Med. Stud. 3, 171-183 (2011).

22. Chiapperino, L. \& Testa, G. The epigenomic self in personalised medicine: between responsibility and empowerment. in Biosocial Matters: Rethinking the Sociology-Biology Relations in the Twenty-First Century (eds. Meloni, M., Williams, S. J. \& Martin, P.) 616 (Wiley-Blackwell, 2016).

23. Heijmans, B. T. \& Mill, J. Commentary: The seven plagues of epigenetic epidemiology. Int. J. Epidemiol. 41, 74-78 (2012).

24. Mill, J. \& Heijmans, B. T. From promises to practical strategies in epigenetic epidemiology. Nat. Rev. Genet. 14, 585-594 (2013).

25. Dupras, C. \& Ravitsky, V. The ambiguous nature of epigenetic responsibility. J. Med. Ethics medethics-2015-103295 (2016). doi:10.1136/medethics-2015-103295

26. Hanson, M. \& Müller, R. Epigenetic inheritance and the responsibility for health in society. Lancet Diabetes Endocrinol. 5, 11-12 (2017).

27. Müller, R. et al. The biosocial genome?: Interdisciplinary perspectives on environmental epigenetics, health and society. EMBO Rep. e201744953 (2017). doi:10.15252/embr.201744953

28. Wilkinson, S. Choosing tomorrow's children: the ethics of selective reproduction. (Clarendon Press, 2010).

29. Thompson, C. Making parents: the ontological choreography of reproductive technologies. (MIT Press, 2005).

30. Savulescu, J. Procreative beneficence: why we should select the best children. Bioethics 15, 
413-426 (2001).

31. Mansfield, B. Folded Futurity: Epigenetic Plasticity, Temporality, and New Thresholds of Fetal Life. Sci. Cult. 1-25 (2017). doi:10.1080/09505431.2017.1294575

32. Warin, M., Moore, V., Zivkovic, T. \& Davies, M. Telescoping the origins of obesity to women's bodies: How gender inequalities are being squeezed out of Barker's hypothesis. Ann. Hum. Biol. 38, 453-460 (2011).

33. Waggoner, M. The Zero Trimester: Pre-Pregnancy Care and the Politics of Reproductive Risk. (University of California Press, 2017).

34. Juengst, E. T., Fishman, J. R., McGowan, M. L. \& Settersten, R. A. Serving epigenetics before its time. Trends Genet. 30, 427-429 (2014).

35. Del Savio, L., Loi Michele \& Stupka Elia. Epigenetics and Future Generations. Bioethics 29, 580-587 (2015).

36. Bode, P. Identity and Non-identity. Intergenerational Justice as a Topic of an Ethics of Epigenetics. in Epigenetics 55-67 (Springer VS, Wiesbaden, 2017).

37. Loi, M. \& Nobile, M. The Moral and Legal Relevance of DOHaD Effects for Pregnant Mothers. in The epigenome and developmental origins of health and disease (ed. Rosenfeld, C. S.) 562 (Academic Press, 2015).

38. Godfrey, K. M., Lillycrop, K. A., Burdge, G. C., Gluckman, P. D. \& Hanson, M. A. Epigenetic Mechanisms and the Mismatch Concept of the Developmental Origins of Health and Disease. Pediatr. Res. 61, 5R-10R (2007).

39. Guibet Lafaye, C. Quelle théorie de la justice pour l'épigénétique? Dialogue 54, 489-517 (2015).

40. Loi, M., Savio, L. D. \& Stupka, E. Social Epigenetics and Equality of Opportunity. Public Health Ethics 6, 142-153 (2013).

41. Marmot, M. Social determinants of health inequalities. The lancet 365, 1099-1104 (2005).

42. Rothstein, M. A., Harrell, H. L. \& Marchant, G. E. Transgenerational epigenetics and environmental justice. Environ. Epigenetics 3, (2017).

43. Huang, J. Y. \& King, N. B. Epigenetics Changes Nothing: What a New Scientific Field Does and Does Not Mean for Ethics and Social Justice. Public Health Ethics 11, 69-81 (2018).

44. Stapleton, G., Schröder-Bäck, P. \& Townend, D. Equity in Public Health: An Epigenetic Perspective. Public Health Genomics 16, 135-144 (2013).

45. Kollar, E. \& Loi, M. Prenatal Equality of Opportunity. J. Appl. Philos. 32, 35-49 (2015).

46. The Palgrave Handbook of Biology and Society. (Palgrave Macmillan, 2018). 